\title{
SEL Hardness Assurance in a Mixed Radiation Field
}

\author{
${ }^{1}$ R. García Alía, ${ }^{1}$ M. Brugger, ${ }^{1}$ S. Danzeca, ${ }^{2}$ V. Ferlet-Cavrois, ${ }^{3}$ C. Frost, ${ }^{4}$ R. Gaillard, ${ }^{1} \mathrm{~J}$. Mekki, \\ ${ }^{5}$ F. Saigné, ${ }^{1} A$. Thornton, ${ }^{1}$ S. Uznanski, ${ }^{5,6}$ F. Worbel \\ ${ }^{1}$ CERN, CH-1211, Genève, Switzerland \\ ${ }^{2}$ European Space Agency, ESTEC, 2200 AG Noordwijk, the Netherlands. \\ ${ }^{3}$ ISIS Facility, Rutherford Appleton Laboratory, Harwell Oxford, Didcot OX11 0QX, U.K. \\ ${ }^{4}$ Consultant in Saint-Arnoult 78730, France \\ ${ }^{5}$ Institut d'Electronique du Sud, Université de Montpellier II/CNRS, 34095 Montpellier Cedex 5, \\ France \\ ${ }^{6}$ Institut Universitaire de France, 75231 Paris Cedex 05, France
}

Keywords: CERN LHC-R2E, CERN East Area, CHARM radiation facility, FLUKA, hardness assurance, high-Z material, Monte Carlo methods, single event latchup (SEL).

\begin{abstract}
This paper explores the relationship between monoenergetic and mixed-field Single Event Latchup (SEL) cross sections, concluding that for components with a very strong energy dependence and highly-energetic environments, test results from monoenergetic or soft mixed-field spectra can significantly underestimate the operational failure rate. We introduce a semi-empirical approach that can be used to evaluate the SEL rate for such environments based on monoenergetic measurements and information or assumptions on the respective sensitive volume and materials surrounding it. We show that the presence of high-Z materials such as tungsten is particularly important in determining the hadron cross section energy dependence for components with relatively large LET thresholds.
\end{abstract}

Presented at:

NSREC 2015 - IEEE Nuclear and Space Radiation Effects Conference

Boston, MA (USA)

July, 2015

Published in:

IEEE Transactions on Nuclear Science, vol. 62, no. 6, pp. 2555-2562, Dec. 2015. 


\title{
SEL Hardness Assurance in a Mixed Radiation Field
}

\author{
Rubén García Alía, Markus Brugger, Salvatore Danzeca, Véronique Ferlet-Cavrois, Christopher Frost, \\ Rémi Gaillard, Julien Mekki, Frédéric Saigné, Adam Thornton, Slawosz Uznanski, Frédéric Wrobel
}

\begin{abstract}
This paper explores the relationship between monoenergetic and mixed-field Single Event Latchup (SEL) cross sections, concluding that for components with a very strong energy dependence and highly-energetic environments, test results from monoenergetic or soft mixed-field spectra can significantly underestimate the operational failure rate. We introduce a semiempirical approach that can be used to evaluate the SEL rate for such environments based on monoenergetic measurements and information or assumptions on the respective sensitive volume and materials surrounding it. We show that the presence of high- $Z$ materials such as tungsten is particularly important in determining the hadron cross section energy dependence for components with relatively large LET thresholds.
\end{abstract}

\section{INTRODUCTION}

Single Event Latchup (SEL) poses a serious threat to microelectronic component reliability not only in the space environment but also for ground level [1] and avionics systems [2]. Therefore, the need of characterizing electronic devices for atmospheric and accelerator applications [3] is crucial.

As stated in the JEDEC standard JESD89A [4] for the case of soft errors such as Single Event Upsets (SEU), the recommended approach to evaluate the Soft Error Rate (SER) at ground level is to test components in neutron spallation sources such as the Los Alamos LANSCE or the TRIUMF NIF facilities [5]. However, the limited availability of such test beams and (notably) their lower fluxes when compared to monoenergetic proton cyclotrons has motivated the development of test standards that partially rely on proton measurements to calculate the ground level SER. In this case, the JEDEC standard recommends performing monoenergetic tests using neutrons at $14 \mathrm{MeV}$ and protons at 50, 100 and $150 \mathrm{MeV}$ (or similar) to derive a fit of the data to an analytical expression (typically, a four parameter Weibull function) and convolute it with the environment of interest to obtain an estimation of the operational failure rate. The reason why the use of neutrons is advised instead of protons below $50 \mathrm{MeV}$ is the

R. García Alía (ruben.garcia.alia@cern.ch), M. Brugger, S. Danzeca, J. Mekki, A. Thornton and S. Uznanski are with CERN, CH-1211, Genève, Switzerland.

V. Ferlet-Cavrois is with the European Space Agency, ESTEC, 2200 AG Noordwijk, The Netherlands.

C. Frost is with the ISIS Facility, Rutherford Appleton Laboratory, Harwell Oxford, Didcot OX11 0QX, United Kingdom

F. Saigné and F. Wrobel are with the Institut d'Electronique du Sud, Université de Montpellier II/CNRS, Place Eugène Batallion - 34095 Montpellier Cedex 5, France. F. Wrobel is also with the Institut Universitaire de France.

R. Gaillard is a consultant in 78730 Saint-Arnoult, France. difference between the nucleon-nucleus cross sections in this energy range.

Otherwise, single monoenergetic measurements can be considered as representative of mixed-field operational environments as well. In fact, the generalized conclusion of a study carried out on a broad range of devices [6] (including SRAMs, FPGAs, microprocessors and SDRAMs) is that monoenergetic proton and neutron beams may be used for SEU testing as an alternative to a spallation neutron source provided results within a factor 2 are acceptable. In the case of neutrons, the test energy required to characterize a device's SEU sensitivity can be as low as $14 \mathrm{MeV}$ [7].

In [6] it is concluded that for purposes of SEL testing for ground-level neutrons, a monoenergetic proton beam in the $100-120 \mathrm{MeV}$ range may be used as a substitute of a neutron spallation beam with agreement expected to be within a factor 2. Likewise is the case for avionics applications, for which standards cite $100 \mathrm{MeV}$ as a sufficient energy to characterize the SEE sensitivity [8]. In [9] however, the warning is given that for components with a strong SEL cross section energy dependence, most test facilities will underestimate the failure rate for the natural atmospheric environment. This was also shown to be the expected scenario through calculations for the high-energy accelerator environment [3], [10] in which the particle energy spectra can be of a very hard (i.e. energetic) character.

In the present study, measurements performed in the VESUVIO beam at ISIS [11] and the CERN High-energy Accelerator Mixed field facility (CHARM) are used in combination with the model we introduced in [10] and applied in [3] in order to quantify the risk of underestimating the natural atmospheric and high-energy accelerator failure rates derived from monoenergetic proton tests, thus providing safety margins to be applied to the experimental results.

In addition, ground-level and avionics failure rates from neutron spallation test results are typically derived by scaling the experimental rate with the respective fluxes above 10 $\mathrm{MeV}$ [4]. This approach ignores the potential effect of the differences in the particle energy spectra of the test and operational environments. However, as shown in [12], [13] the SEL, SEU and MBU cross section energy dependence can be significantly impacted by the presence of tungsten near the relevant sensitive volumes, notably for components with high critical charges. Quantifying the risk related to the impact of the spectral hardness in atmospheric and high energy accelerator applications is the main objective of the work we present here. 
Likewise, according to the available standards, variations in the atmospheric neutron flux with respect to the atmospheric depth, the geomagnetic cutoff rigidity and the solar modulation can be accounted for by scaling the reference spectrum (defined in New York city outdoors at sea level and at a time of average solar activity) in terms of intensity, but do not consider variations in the particle energy spectra. In the present work, we show the implications of the different spectral hardnesses of a broad range of experimental and operational environments on the SEL hardness assurance.

\section{HARDNESS ANALYSIS OF EXPERIMENTAL AND OPERATIONAL ENVIRONMENTS}

The test data we present in this work were collected at two experimental facilities: the VESUVIO beam at ISIS [11] and the CHARM facility at CERN. In both cases, the radiation environment is a result of the spallation products of a highenergy proton beam with a heavy target.

At VESUVIO, neutrons are generated through the interaction of a $800 \mathrm{MeV}, 200 \mu \mathrm{A}$ proton beam produced in bunches in a synchrotron with a spallation source. The protons are delivered to the spallation target in two $100 \mathrm{~ns}$ long pulses with a frequency of $50 \mathrm{~Hz}$. The neutron beamline is at $60^{\circ}$ with respect to the initial proton beam and the neutron flux obtained above $10 \mathrm{MeV}$ for nominal operation conditions is $\sim 5.8 \cdot 10^{4} \mathrm{n} / \mathrm{cm}^{2} / \mathrm{s}$. The neutron spectrum is calculated using the MCNPX Monte Carlo simulation tool and was benchmarked against Time-Of-Flight (TOF) measurement performed with various detectors [14]. In addition, it is to be noted that a new neutron beam (ChipIR) will soon be available at ISIS providing larger intensities and energies than what is currently available in VESUVIO.

At CHARM, the mixed radiation field is generated through the interaction of a $24 \mathrm{GeV}$ proton beam from CERN's Proton Synchrotron (PS) with a $50 \mathrm{~cm}$ long, $8 \mathrm{~cm}$ in diameter metal target. Different target materials are available in order to vary the mixed-field intensity for a given incident proton intensity within a certain margin. The protons impinge the target in spills of roughly $3 \cdot 10^{11}$ protons lasting $300 \mathrm{~ms}$. The total cycle of the PS typically lasts between 30 and 40 s, during which CHARM receives between 1 and 6 spills. The proton intensity is measured using calibrated Secondary Emission Counters (SECs) and an ionization chamber. The mixed-field generated through the interaction of the primary $24 \mathrm{GeV}$ beam and the target is simulated using the FLUKA Monte Carlo code [15]-[17]. The quantities scored in such simulations include particle fluxes, $1 \mathrm{MeV}$ neutron equivalent fluxes, TID values and particle energy spectra for the different test locations of interest.

From a particle generation point of view, the fact that the proton energy at CHARM is two orders of magnitude larger than that utilized in cyclotron neutron spallation facilities results in an increased production yield and energy transfer to the secondary hadrons. In addition, pions, kaons and other hadrons are generated in a significant proportion, unlike at several hundred MeVs. Otherwise, whereas in neutron spallation facilities charged particles are removed from the beam through absorbers and magnetic elements, in the case of CHARM these are kept in the environment, which is therefore composed not only of neutrons but also of pions, protons and kaons. Other type of particles such as electrons, positrons, photons, muons, etc. are not explicitly considered in this work as their contribution to SEL is negligible, however they of course add to the Total Ionizing Dose (TID) received by the Devices Under Test.

Likewise, whereas standard spallation facilities typically have specific beam lines for neutrons, at CHARM a large volume surrounding the target is available for SEE testing. This enables on the one hand the testing of bulky systems which can as a whole be exposed to radiation, and on the other a broad range of possible fluxes and particle energy spectra depending on the exact test location. In fact, 19 standard test locations (here referred to as TLs) have been defined in CHARM as can be seen in the horizontal cut in Fig. 1. They are numbered from 1 through 19 starting from the bottom left of the plot, and cover angles with respect to the beam from $135^{\circ}$ to directly downstream the target $\left(0^{\circ}\right)$. Test data presented in this paper were obtained in TLs $1,3,7,14,16$ and 17 , as marked in the figure. In addition, four movable shielding plates, two of $40 \mathrm{~cm}$ concrete and two of $40 \mathrm{~cm}$ iron are available and can be remotely introduced in the facility in order to further alter the mixed-fields depending on the desired user's test parameters. For the results we present here, tests were performed without shielding between the target and the test locations for positions $1,7,14,16$ and 17 , and with a shielding configuration of $80 \mathrm{~cm}$ of concrete and $80 \mathrm{~cm}$ of iron at test locations 3 and 7 . In order to distinguish them from the non-shielded cases, such locations are marked with a star superscript.

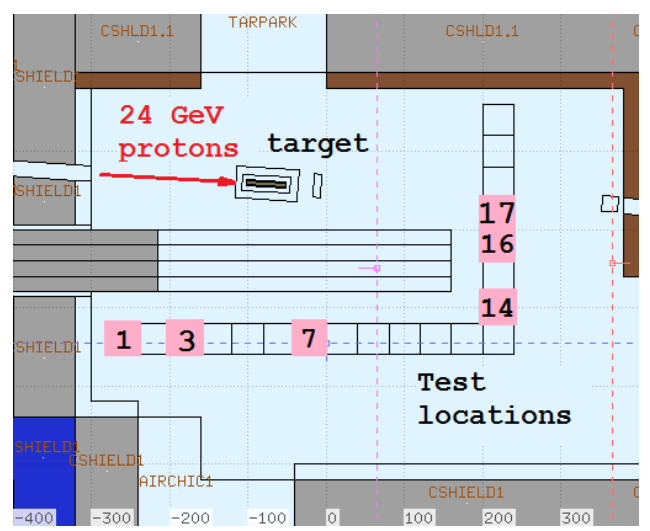

Fig. 1: Horizontal cut of the CHARM geometry as implemented in FLUKA showing the proton beam, spallation target and test locations used to collect the experimental data presented. In this configuration, there is no shielding between the target and the test locations, however measurements at test location 7 were performed both without shielding and with all four blocks placed inside. The shielded configuration was also used for test location 3 .

In order to compare the different environments involved in the measurements we will later present, the normalized differential hadron energy spectra above $20 \mathrm{MeV}$ for VESUVIO and some of the TLs used at CHARM are plotted in Fig. 2 together with those of ChipIR (only based on calculations, 
still to be calibrated). Several operational environments are included as well. The CHARM spectra were extracted using FLUKA, whereas the atmospheric cases were retrieved using the QARM online tool [18] for the Geneva geographic coordinates $\left(46^{\circ} \mathrm{N}, 6^{\circ} \mathrm{E}, 375 \mathrm{~m}\right.$ altitude) and solar maximum conditions. Results for other altitudes (i.e. 12 and $20 \mathrm{~km}$ ) are extracted using the same latitude and longitude. For VESUVIO and ChipIR, the spectra were obtained through MCNPX simulations and provided to us the facility experts.

The fluxes are normalized to the integral hadron flux above $20 \mathrm{MeV}$, defined as High Energy Hadron (HEH) flux and here represented as $\Phi_{\mathrm{HEH}}$. As can be seen, VESUVIO and TL1 of CHARM exhibit the softest spectra, with the groundlevel, TL7 at CHARM and ChipIR being of an intermediate character, and TL 16 and 17 at CHARM and the $20 \mathrm{~km}$ having the hardest nature. It is to be noted that, whereas the VESUVIO and ChipIR spectra are composed solely of neutrons, the ground-level and $20 \mathrm{~km}$ altitude also include protons, and at CHARM a broader range of hadrons (neutrons, protons, pions, kaons, etc.) are present. As to what regards the HEH fluxes during the different tests, at CHARM the maximum value was roughly $2.5 \cdot 10^{6} \mathrm{~cm}^{-2} \mathrm{~s}^{-1}$ for the downstream locations, with lower intensities for the lateral (i.e. 1, 3 and 7 as shown in Fig. 1) and (notably) shielded locations. At VESUVIO, the average test flux was roughly two orders of magnitude smaller than the maximum value at CHARM.

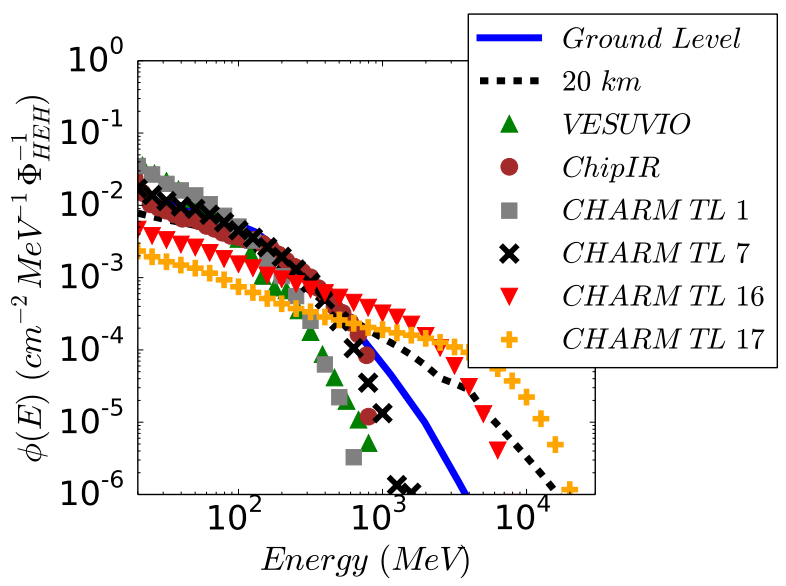

Fig. 2: Differential hadron spectra for different operational and experimental environments normalized to the respective integral $\mathrm{HEH}$ fluxes.

In order to further characterize and compare the hadron energy spectra of the different environments, hardness energies at different percentages can be defined as the energies above which a certain proportion of the HEH remains. For instance, we introduce the $50 \%(10 \%)$ hardness energy as the energy above which $50 \%(10 \%)$ of the total HEH flux is found, and represented hereafter as $\mathrm{H}_{50 \%}\left(\mathrm{H}_{10 \%}\right)$. Whereas this Figureof-Merit does not uniquely define the spectra, it is useful to describe its hardness through a reduced set of values. The respective values for the spectra shown in Fig. 2 can be found in Table I together with other relevant experimental and operational radiation environments. The LHC experiment example refers to hadrons scored using FLUKA in a cylindrical region of $20 \mathrm{~cm}$ in radius and $3.0 \mathrm{~m}$ in length centered on the interaction point of the ATLAS inner detector.

TABLE I: Hardness energies as defined in the text for different experimental and operational environments. CHARM locations marked with an asterisk refer to test conditions in which the full movable shielding was placed inside the irradiation area. Hardness energy values may slightly differ from those shown in [3] for the same spectra due to the use of different sources to obtain the spectra, updated simulations, different binning, etc.

\begin{tabular}{c|c|c}
\hline Env. & $\mathrm{H}_{50 \%}(\mathrm{MeV})$ & $\mathrm{H}_{10 \%}(\mathrm{MeV})$ \\
\hline \hline VESUVIO & 45 & 150 \\
ChipIR & 120 & 410 \\
CHARM (3*) & 70 & 170 \\
CHARM (1) & 65 & 175 \\
CHARM (7*) & 75 & 220 \\
CHARM (7) & 100 & 370 \\
CHARM (14) & 280 & $1.1 \mathrm{GeV}$ \\
CHARM (16) & 700 & $2.9 \mathrm{GeV}$ \\
CHARM (17) & $2.6 \mathrm{GeV}$ & $9.7 \mathrm{GeV}$ \\
\hline Ground-level & 140 & 540 \\
12 km & 150 & $1.0 \mathrm{GeV}$ \\
20 km & 200 & $3.2 \mathrm{GeV}$ \\
LHC Experiment & 890 & $3.8 \mathrm{GeV}$ \\
Interplanetary & $1.2 \mathrm{GeV}$ & $5.6 \mathrm{GeV}$ \\
\hline
\end{tabular}

\section{Mixed-Field Test Results}

Four different commercial SRAM components were used as a test sample. Their respective characteristics are shown in Table II together with the naming convention used in the present paper, selected in order to be consistent with that used in [3], [10]. In order to characterize their SEL response, the memories were powered and their current consumption was monitored with a frequency of $1 \mathrm{~Hz}$ through a LabVIEW interface. When the current consumption exceeded the nominal value (ranging from $2 \mathrm{~mA}$ for SRAM D to $32 \mathrm{~mA}$ for SRAM A) by a certain margin (typically $50 \mathrm{~mA}$ ), a power cycle was performed. During the time in which the components remained in the high current state, the current supply was limited to roughly $80 \mathrm{~mA}$ in order to avoid permanent damage of the components. The total detection plus power cycle time was $6 \mathrm{~s}$, which was accordingly subtracted in the fluence calculation in order to discard the dead time of the devices. In all case, the latter was maintained below $20 \%$ of the total beam time. The memory pattern was the preferred one when the components were powered and their functionality in terms of write/read operations was verified both before and after the radiation tests. In addition, the SEL rate was monitored throughout the tests evidencing that it did not significantly evolve with time, thus no TID or latent effects due to the repeated SELs could be identified. All four components were powered at $3.3 \mathrm{~V}$ and measurements were performed at room temperature.

TABLE II: Main characteristics of the components tested and their identification name as considered in the present work.

\begin{tabular}{c|c|c|c|c}
\hline ID & Part Numbers & Manufacturer & $\begin{array}{c}\text { Tech. } \\
(\mathrm{nm})\end{array}$ & $\begin{array}{c}\text { Date } \\
\text { Code }\end{array}$ \\
\hline \hline SRAM A & IS61LV5128AL-10TLI & ISSI & 180 & 1247 \\
SRAM C & AS7C34098A-10TCN & Alliance & 200 & $1205,1210,1339$ \\
SRAM D & K6R4016V1D-TC10 & Samsung & 180 & 413,922 \\
SRAM F & BS62LV1600EIP55 & Brilliance & 180 & 11254 \\
\hline
\end{tabular}


The cross sections for the respective mixed-field environments were derived by dividing the SEL count by the effective HEH fluence (i.e. excluding the detection and power cycle dead time periods). For this reason, we will refer to them as experimental mixed-field HEH cross sections, here denoted as $\sigma_{\text {exp. }}^{*}$, where the star is used to distinguish these cross sections from the monoenergetic ones. In all cases, more than 50 SEL events were collected per component type and test conditions. At CHARM, depending on the device sensitivity and test flux, several components (up to 16) were tested simultaneously in boards hosting 8 components each. In all cases the flux was considered to be homogeneous within $\pm 10 \%$ throughout the surface covered by the board. In addition, the sensitivity deviations among individual components of the same reference were measured to be smaller than those related to the test facility dosimetry (considered as 10\%) and the error count, even for components of different date codes. However, we consider a worst-case $20 \%$ sensitivity variability among different individual components of the same reference. Therefore, the uncertainly on the cross section values takes into account (i) a $10 \%$ error in the facility dosimetry (ii) a $10 \%$ error associated to the mixed-field homogeneity and size of the scoring volumes in the FLUKA simulation (iii) a $20 \%$ spread in the individual sensitivities and (iv) the 2-sigma error associated to the number of SEL counts.

The mixed-field HEH results for the four components considered in the VESUVIO and CHARM environments are shown in Table III. In order to graphically compare the results, the cross sections normalized to the TL1 value are shown in Fig. 3 for SRAMs A, C and D as a function of the respective $10 \%$ hardness energies shown in Table I. As can be seen, two clearly distinct behaviors can be identified: whereas for SRAM A the dependency of the cross section with $\mathrm{H}_{10 \%}$ is relatively weak (less than a factor 2 increase between $175 \mathrm{MeV}$ and 1.1 $\mathrm{GeV}$ ) for SRAMs $\mathrm{C}$ and $\mathrm{D}$, the impact of the spectral hardness on the SEL rate is significant (over a factor 10 when $\mathrm{H}_{10 \%}$ augments from $175 \mathrm{MeV}$ to $1.1 \mathrm{GeV}$ and roughly a factor 100 if we consider the difference between $175 \mathrm{MeV}$ and $9.7 \mathrm{GeV}$ ).

TABLE III: Measured SEL cross sections in units of $\mathrm{cm}^{2} /$ device for the different SRAMs and environments considered. The relative uncertainties are not explicitly shown but are below $37 \%$ in all cases.

\begin{tabular}{c|c|c|c|c|c}
\hline Env. & $\begin{array}{c}\mathrm{H}_{10 \%} \\
(\mathrm{MeV})\end{array}$ & SRAM A & SRAM C & SRAM D & SRAM F \\
\hline \hline VESUVIO & 150 & $5.7 \cdot 10^{-9}$ & - & - & - \\
CHARM (3*) & 170 & $8.8 \cdot 10^{-9}$ & - & - & - \\
CHARM (1) & 175 & $9.8 \cdot 10^{-9}$ & $7.8 \cdot 10^{-11}$ & $5.0 \cdot 10^{-11}$ & - \\
CHARM (7*) & 220 & $1.2 \cdot 10^{-8}$ & - & - & $1.3 \cdot 10^{-7}$ \\
CHARM (7) & 355 & - & $1.7 \cdot 10^{-10}$ & $2.6 \cdot 10^{-10}$ & - \\
CHARM (14) & $1.1 \mathrm{GeV}$ & $1.7 \cdot 10^{-8}$ & $6.3 \cdot 10^{-10}$ & $9.8 \cdot 10^{-10}$ & - \\
CHARM (16) & $2.9 \mathrm{GeV}$ & - & $1.6 \cdot 10^{-9}$ & $2.3 \cdot 10^{-9}$ & - \\
CHARM (17) & $9.7 \mathrm{GeV}$ & - & $5.3 \cdot 10^{-9}$ & $6.8 \cdot 10^{-9}$ & - \\
\hline
\end{tabular}

In the following section, the dependency of the mixed-field cross section on the spectral hardness of the environment will be analyzed and correlated with the respective monoenergetic responses both from an experimental and a simulation point of view.

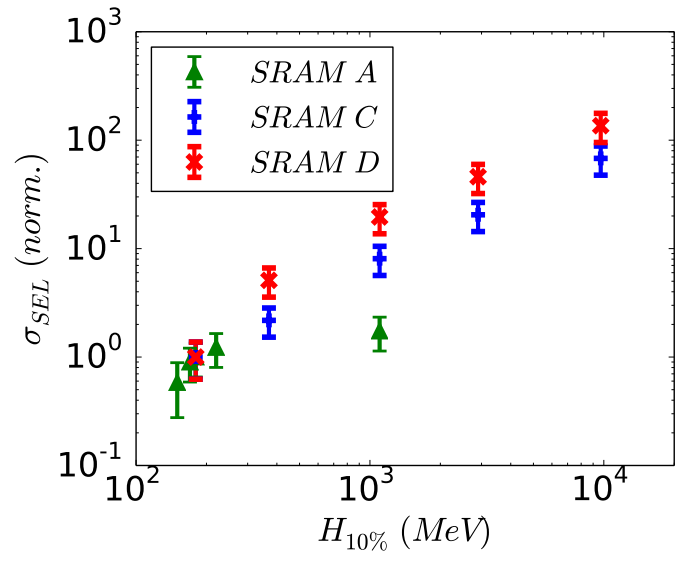

Fig. 3: Mixed-field HEH cross sections for the different components and environments considered normalized to the CHARM (1) value.

\section{COMPARISON WITH EXPECTED MIXED-FIELD SEL RATE}

As suggested in the JEDEC standard [4] for SEU, the mixed-field SEL cross section $\sigma_{\text {calc. }}^{*}$ can be extracted by folding the analytic fits to the monoenergetic cross section $\sigma(\mathrm{E})$ with the differential HEH spectrum $\phi(\mathrm{E})$ as shown in Eq. 1, where the flux considered is that of all hadrons above $20 \mathrm{MeV}$, as is the standard case in the high-energy accelerator context [19]. It is to be noted that in the groundlevel application context, the neutron flux above $10 \mathrm{MeV}$ is normally considered instead. Typically, the analytic fit to the experimental data is performed using a four-parameter Weibull function of the type shown in Eq. 2.

$$
\begin{gathered}
\sigma_{\text {calc. }}^{*}=\frac{\int_{\mathrm{E}_{\min }}^{\mathrm{E}_{\max }} \sigma(\mathrm{E}) \cdot \phi(\mathrm{E}) \mathrm{dE}}{\int_{20 \mathrm{MeV}}^{\mathrm{E}_{\max }} \phi(\mathrm{E}) \mathrm{dE}} \\
\sigma(\mathrm{E})=\sigma_{\text {sat }} \cdot\left(1-\exp \left(-\left(\mathrm{E}-\mathrm{E}_{\mathrm{o}}\right) / \mathrm{W}\right)^{\mathrm{s}}\right)
\end{gathered}
$$

By using a single cross section and flux in Eq. 1 we assume that all hadrons in the mixed-field are equally efficient in inducing SELs. In this case, the vast majority of monoenergetic results were extracted using proton beams.

We performed monoenergetic SEL measurements on the four SRAMs studied in this paper at different facilities. As detailed in [3] we used the Proton Irradiation Facility (PIF) at the Paul Scherrer Institute [20] for proton measurements between 30 and $230 \mathrm{MeV}$ and the Proton Irradiation Facility (PIF) at TRIUMF [21] to cover the $230-480 \mathrm{MeV}$ range. In addition, a $300 \mathrm{MeV}$ quasi-monoenergetic neutron measurement was performed on SRAM F at RCNP in Osaka, Japan (details of the test and analysis will be provided in a future publication).

\section{A. Saturated cross sections at $230 \mathrm{MeV}$}

As a first approach, we will assume that the SEL responses of the components considered saturate at $230 \mathrm{MeV}$ (which is the maximum proton energy available at PSI). This energy is above the maximum recommended in the JEDEC standard 
$(150 \mathrm{MeV})$ and from a nuclear reaction point of view is considered to be an energy for which SEE cross sections in silicon are saturated [22], [23].

SEL cross section results for SRAMs A and F are shown in Figs. 4 and 5 together with the fit to a four-parameter Weibull function of the type shown in Eq. 2 and with the values presented in Table IV. All monoenergetic measurements plotted in this paper consider error bars that include: (i) a $10 \%$ uncertainty in the fluence measurement (ii) a $20 \%$ part-to-part variability (iii) the 2 -sigma counting error.

TABLE IV: Four-parameter Weibull fit to the SEL cross section for SRAMs A and F performed using a non-linear least squares fit.

\begin{tabular}{c|c|c}
\hline Parameter & SRAM A & SRAM F \\
\hline \hline$\sigma_{\text {sat }}\left(\mathrm{cm}^{2}\right)$ & $3.09 \cdot 10^{-8}$ & $3.13 \cdot 10^{-7}$ \\
$\mathrm{E}_{\mathrm{o}}(\mathrm{MeV})$ & 17.2 & 27.0 \\
$\mathrm{~W}(\mathrm{MeV})$ & 139 & 57.2 \\
$\mathrm{~s}$ & 1.36 & 1.16 \\
\hline
\end{tabular}

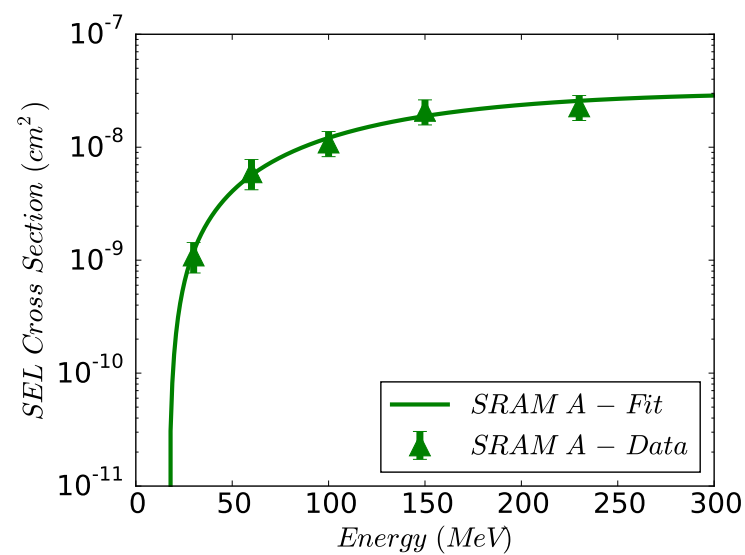

Fig. 4: SEL cross section for SRAM A along with the respective Weibull fit.

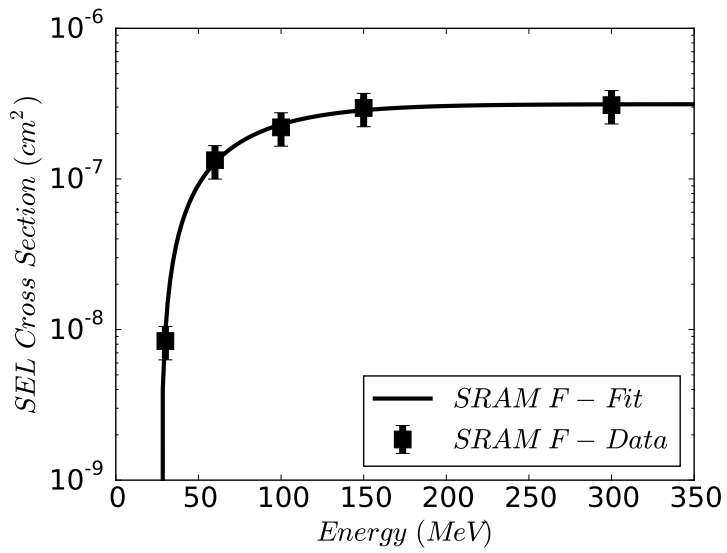

Fig. 5: SEL cross section for SRAM F along with the respective Weibull fit.

Once the fits are defined, they can be used in combination with the energy spectrum of the radiation environment in order to extract the expected mixed-field cross section. This was performed for SRAM A and the different environments in which it was tested, yielding the results shown in Table V. The ratio between the calculated and experimental cross section values is also shown. As can be seen, all calculated cross section values are within $\pm 31 \%$ of the experimental value, which can be considered as a highly satisfactory agreement when taking into account the uncertainty associated to both the measurements and the calculation method.

TABLE V: Calculated mixed-field cross section and ratio to experimental value for SRAMs A and F.

\begin{tabular}{c|c|c|c|c|c}
\hline \multirow{2}{*}{ Env. } & \multirow{2}{*}{$\begin{array}{c}\mathrm{H}_{10 \%} \\
(\mathrm{MeV})\end{array}$} & $\begin{array}{c}\sigma_{\text {calc. }}^{*} \\
\left(\mathrm{~cm}^{2}\right)\end{array}$ & $\frac{\sigma_{\text {calc. }}^{*}}{\sigma_{\text {exp. }}^{*}}$ & $\begin{array}{c}\sigma_{\text {calc. }}^{*} \\
\left(\mathrm{~cm}^{2}\right)\end{array}$ & $\frac{\sigma_{\text {calc. }}^{*}}{\sigma_{\text {exp. }}^{*}}$ \\
\cline { 3 - 6 } & 145 & $6.6 \cdot 10^{-9}$ & $\mathbf{1 . 1 6}$ & - & - \\
\hline VESUVIO & 170 & $9.2 \cdot 10^{-9}$ & $\mathbf{1 . 0 5}$ & - & - \\
CHARM (3*) & 175 & $8.3 \cdot 10^{-9}$ & $\mathbf{0 . 8 5}$ & - & \\
CHARM (1) & 220 & $1.1 \cdot 10^{-8}$ & $\mathbf{0 . 9 2}$ & $1.7 \cdot 10^{-7}$ & $\mathbf{1 . 3 1}$ \\
CHARM (14) & $1.1 \mathrm{GeV}$ & $2.2 \cdot 10^{-8}$ & $\mathbf{1 . 2 9}$ & - & - \\
\hline
\end{tabular}

For SRAMs C and D, the calculation approach is different owing to their relatively low cross section, which meant it was not possible to obtain a statistically meaningful number of counts $(>50)$ for energies below $150 \mathrm{MeV}$. For this reason, we use the output of the Monte Carlo based semi-empirical SEL model introduced in [10] and that will be briefly covered in subsection IV-B to estimate the cross section in the 30$100 \mathrm{MeV}$ range. As was performed for SRAMs A and F, we initially assume that the SEL cross section saturates at the maximum energy tested for at PSI (i.e. $230 \mathrm{MeV}$ ). The respective simulated data points are shown in Figs. 6 and 7 together with the measurements at 150 and $230 \mathrm{MeV}$. The fits to the simulated data assuming a saturation at $230 \mathrm{MeV}$ are also included. The corresponding fit parameters are shown in Table VI.

TABLE VI: Two-parameter Weibull fit to the SEL cross section for SRAMs $C$ and D performed using a non-linear least squares fit. $E_{O}$ was set to $20 \mathrm{MeV}$ as the value obtained from the fit was unphysical, and $\sigma_{\text {sat }}$ was fixed $20 \%$ above the $230 \mathrm{MeV}$ value.

\begin{tabular}{c|c|c}
\hline Parameter & SRAM C & SRAM D \\
\hline \hline$\sigma_{\text {sat }}\left(\mathrm{cm}^{2}\right)$ & $4.0 \cdot 10^{-10}$ & $5.0 \cdot 10^{-10}$ \\
$\mathrm{E}_{\mathrm{o}}(\mathrm{MeV})$ & 20 & 20 \\
$\mathrm{~W}(\mathrm{MeV})$ & 156 & 157 \\
$\mathrm{~s}$ & 1.93 & 1.74 \\
\hline
\end{tabular}

We then use the fits to the data for SRAM C and D to derive the expected mixed-field SEL cross section in the environments of interest using Eq. 1. The resulting values are shown in Table VII both in absolute terms and relative to the experimental value. As can be seen, for TL1 and 7, the expected results are within $\pm 27 \%$ of the experimental values except for SRAM D in TL1, for which the calculation overestimates the measurement by a factor 2 . We attribute this overestimation to the importance of the simulated $30 \mathrm{MeV}$ point (see Fig. 7) in this soft environment. For an environment of an intermediate hardness such as TL7, the energies in the 100-200 MeV range (for which experimental data are available for SRAM D) play a stronger role. 


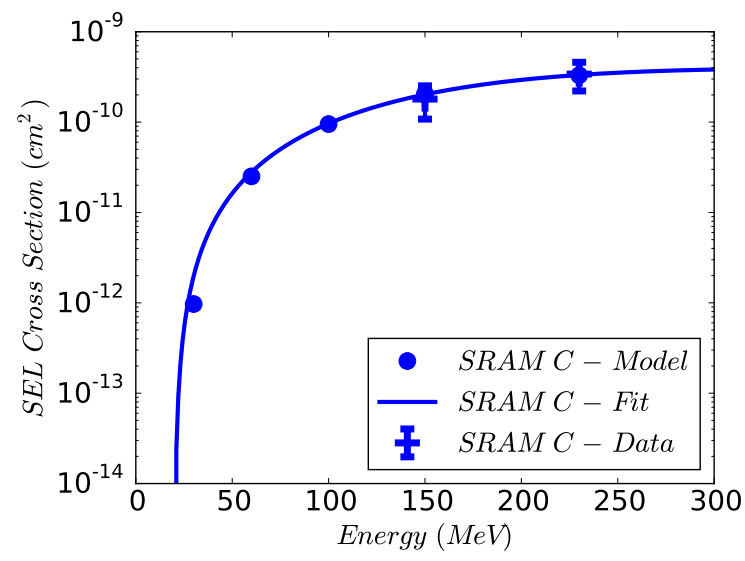

Fig. 6: SEL cross section for SRAM C assuming a saturation just above $230 \mathrm{MeV}$ along with the respective Weibull fit.

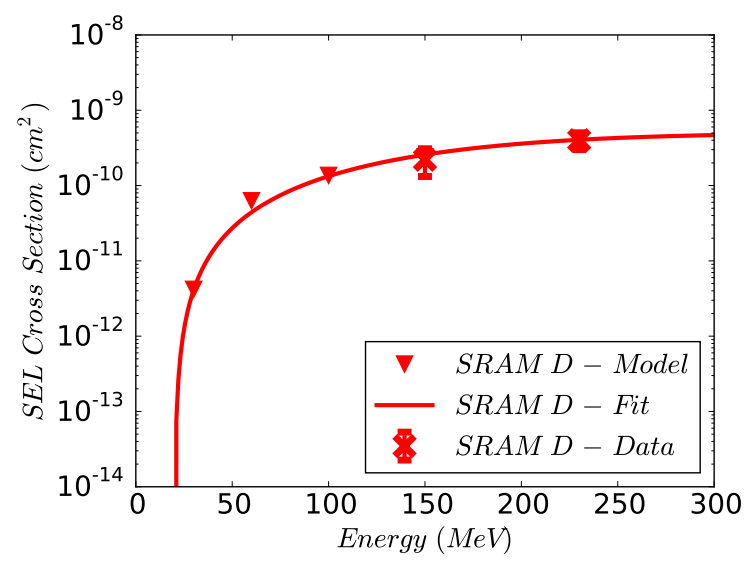

Fig. 7: SEL cross section for SRAM D assuming a saturation just above $230 \mathrm{MeV}$ along with the respective Weibull fit.

However, as the hardness of the environment increases, the calculations tend to systematically underestimate the measurements (by a factor $\sim 2$ for TL14, a factor $\sim 5$ for TL16 and a factor $\sim 15$ for TL17). As predicted in [3], we attribute this behavior to the fact that the cross section is not actually saturated at $230 \mathrm{MeV}$ but is instead expected to increases significantly up to an energy of $3 \mathrm{GeV}$. This increase is driven by the enhanced fission cross section in tungsten elements near the SEL sensitive volume. A Scanning Electron Microscope (SEM) horizontal image of the tungsten elements in a cell of SRAM C is shown in Fig. 8, corresponding to a volume of $\sim 0.5 \mu \mathrm{m}^{3}$ per cell. This value was in fact predicted in [10] through a fit of the Monte Carlo SEL model to the experimental data in the $100-230 \mathrm{MeV}$ range. The impact of the energy dependency derived from the high- $Z$ elements on the mixed-field cross sections (initially introduced in [12]) will be quantified in the following subsection.

\section{B. High-Z SEL model for cross section energy dependence}

In [10] we introduced a semi-empirical SEL cross section estimation approach in which technological input parameters,
TABLE VII: Calculated mixed-field cross section and ratio to experimental value for SRAMs C and D assuming a saturated cross section at $230 \mathrm{MeV}$.

\begin{tabular}{c|c|c|c|c|c}
\hline \multirow{2}{*}{ Env. } & \multirow{2}{*}{$\begin{array}{c}\mathrm{H}_{10 \%} \\
(\mathrm{MeV})\end{array}$} & $\begin{array}{c}\sigma_{\text {sim. }}^{*} \text { SRAM C } \\
\left(\mathrm{cm}^{2}\right)\end{array}$ & $\frac{\sigma_{\text {sim. }}^{*}}{\sigma_{\text {exp. }}^{*}}$ & $\begin{array}{c}\sigma_{\text {sim. }}^{*} \\
\left(\mathrm{~cm}^{2}\right)\end{array}$ & $\frac{\sigma_{\text {sim. }}^{*}}{\sigma_{\text {exp. }}^{*}}$ \\
\hline CHARM (1) & 175 & $7.6 \cdot 10^{-11}$ & $\mathbf{0 . 9 7}$ & $1.0 \cdot 10^{-10}$ & $\mathbf{2 . 0}$ \\
CHARM (7) & 220 & $1.5 \cdot 10^{-10}$ & $\mathbf{0 . 8 8}$ & $1.9 \cdot 10^{-10}$ & $\mathbf{0 . 7 3}$ \\
CHARM (14) & $1.1 \mathrm{GeV}$ & $2.6 \cdot 10^{-10}$ & $\mathbf{0 . 4 1}$ & $3.2 \cdot 10^{-10}$ & $\mathbf{0 . 5 1}$ \\
CHARM (16) & $2.9 \mathrm{GeV}$ & $3.1 \cdot 10^{-10}$ & $\mathbf{0 . 1 9}$ & $3.9 \cdot 10^{-10}$ & $\mathbf{0 . 1 7}$ \\
CHARM (17) & $9.7 \mathrm{GeV}$ & $3.6 \cdot 10^{-10}$ & $\mathbf{6 . 8} \cdot \mathbf{1 0}^{-2}$ & $4.5 \cdot 10^{-10}$ & $\mathbf{6 . 6} \cdot \mathbf{1 0}^{-\mathbf{2}}$ \\
\hline
\end{tabular}

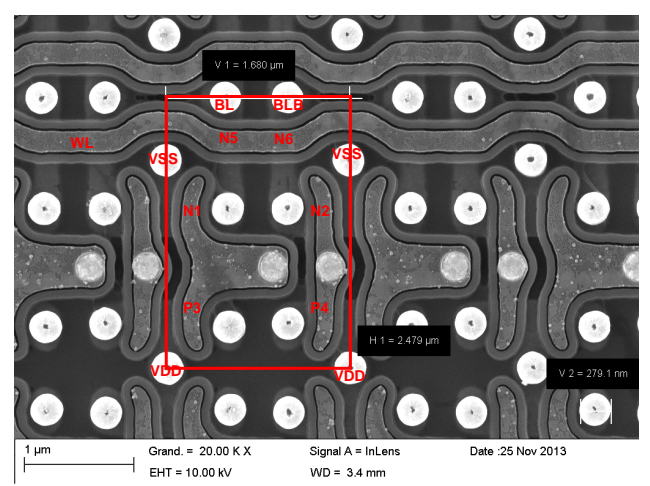

Fig. 8: Horizontal section of SRAM C at polysilicon level showing the vias in white (i.e. tungsten). $\mathrm{N} 1+\mathrm{P} 3$ and $\mathrm{N} 2+\mathrm{P} 4$ form the crosscoupled inverters of the cell whereas $\mathrm{N} 5$ and $\mathrm{N} 6$ are the access transistors.

the heavy ion (HI) cross section response and the monoenergetic proton data in the $100-230 \mathrm{MeV}$ are used in combination with Monte Carlo simulations to calibrate a model and apply it to hadrons and energies typically not accessible in standard radiation facilities. The different steps, detailed in [10], can be summarized as follows:

(i) we simulate the energy deposition for a $100 \mathrm{MeV}$ proton beam on a standard SRAM SEL sensitive surface and surroundings considering different sensitive depths;

(ii) we obtain the simulated proton cross section as a function of the thickness by folding the different energy deposition distributions with the HI response and select the SV depth value that best fits the $100 \mathrm{MeV}$ proton experimental value;

(iii) Steps (i) and (ii) are repeated for $230 \mathrm{MeV}$ protons and the tungsten volume near the SV as a fitting parameter;

(iv) We use the calibrated model to estimate the cross section for larger energies not easily accessible experimentally but still relevant in highly energetic environments.

As shown in [3], this model was successful in predicting both the tungsten volume per cell in SRAMs C and D (roughly $\left.0.5 \mu \mathrm{m}^{3}\right)$ as well as their cross section increase at $480 \mathrm{MeV}$.

Therefore, we use this SEL model to extract the expected SEL cross section considering the effect of tungsten elements near the relevant SVs for those SRAMs in which it is expected to play an important role. The respective simulated cross sections and fits for SRAMs $\mathrm{C}$ and D are shown in Figs. 9 and 10 together with the data in the $150-480 \mathrm{MeV}$ range. The respective fit parameters are included in Table VIII. The 230 $\mathrm{MeV}$ saturated fits used in subsection IV-A are also included in the figures, clearly showing that (i) the data above $230 \mathrm{MeV}$ 
is not compatible with saturation and (ii) the cross section is expected to increase by over a factor 10 between $230 \mathrm{MeV}$ and $3 \mathrm{GeV}$.

TABLE VIII: Weibull fit parameters to the SEL cross section for SRAMs C and D up to $30 \mathrm{GeV}$ performed considering two parameters (W and s) and a non-linear least squares fit. The threshold energy was fixed to $25 \mathrm{MeV}$ and the saturation cross section was selected compatible with the constant value between 3 and $30 \mathrm{GeV}$.

\begin{tabular}{c|c|c}
\hline Parameter & SRAM C & SRAM D \\
\hline \hline$\sigma_{\text {sat }}\left(\mathrm{cm}^{2}\right)$ & $7.5 \cdot 10^{-9}$ & $7.5 \cdot 10^{-9}$ \\
$\mathrm{E}_{\mathrm{o}}(\mathrm{MeV})$ & 25 & 25 \\
$\mathrm{~W}(\mathrm{MeV})$ & $2.21 \cdot 10^{3}$ & $3.00 \cdot 10^{3}$ \\
$\mathrm{~s}$ & 1.41 & 1.01 \\
\hline
\end{tabular}

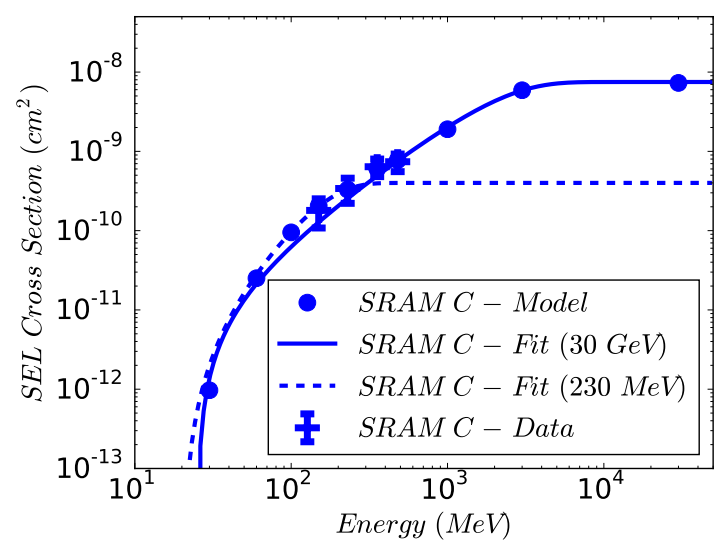

Fig. 9: Simulated SEL cross section for SRAM C according to the high- $\mathrm{Z}$ model introduced in the text along with the respective Weibull fit, the experimental data and the fit considering saturation just above $230 \mathrm{MeV}$.

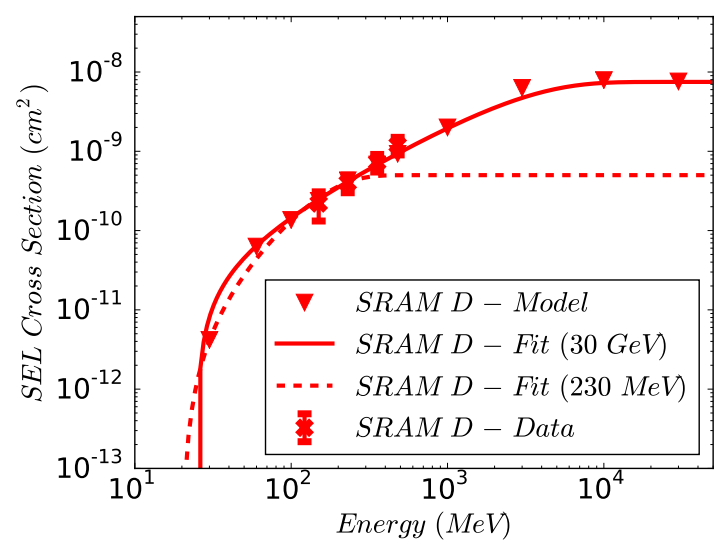

Fig. 10: Simulated SEL cross section for SRAM D according to the high- $\mathrm{Z}$ model introduced in the text along with the respective Weibull fit, the experimental data and the fit considering saturation just above $230 \mathrm{MeV}$.

By using the fits to the simulated data including the high$\mathrm{Z}$ material effect (i.e. those considering the increase with energy up to $30 \mathrm{GeV}$ ) we extract the expected mixed-field cross sections, which are shown in Table IX for the hard environments (as results for the softer ones are hardly affected by the cross section increase above $230 \mathrm{MeV}$ ).

TABLE IX: Calculated mixed-field cross section and ratio to experimental value for SRAMs C and D considering the high-Z SEL model for the energy dependence above $230 \mathrm{MeV}$.

\begin{tabular}{c|c|c|c|c|c}
\hline \multirow{2}{*}{ Env. } & \multirow{2}{*}{$\begin{array}{c}\mathrm{H}_{10 \%} \\
(\mathrm{MeV})\end{array}$} & \multicolumn{2}{|c|}{ SRAM C } & \multicolumn{2}{c}{ SRAM D } \\
\cline { 3 - 6 } & $\left(\mathrm{cm}^{2}\right)$ & $\frac{\sigma_{\text {sim. }}^{*}}{\sigma_{\text {exp. }}^{*}}$ & $\begin{array}{c}\sigma_{\text {sim. }}^{*} \\
\left(\mathrm{~cm}^{2}\right)\end{array}$ & $\frac{\sigma_{\text {sim. }}^{*}}{\sigma_{\text {exp. }}^{*}}$ \\
\hline \hline CHARM (14) & $1.1 \mathrm{GeV}$ & $7.8 \cdot 10^{-10}$ & $\mathbf{1 . 2 4}$ & $8.8 \cdot 10^{-10}$ & $\mathbf{0 . 9 0}$ \\
CHARM (16) & $2.9 \mathrm{GeV}$ & $2.1 \cdot 10^{-9}$ & $\mathbf{1 . 3 1}$ & $1.9 \cdot 10^{-9}$ & $\mathbf{0 . 8 3}$ \\
CHARM (17) & $9.7 \mathrm{GeV}$ & $4.4 \cdot 10^{-9}$ & $\mathbf{0 . 8 3}$ & $4.0 \cdot 10^{-9}$ & $\mathbf{0 . 5 9}$ \\
\hline
\end{tabular}

As can be seen, by using the high-Z SEL model, the calculated results are in agreement with the measurements within $\pm 40 \%$ (and mostly within $\pm 30 \%$ ) which can be regarded as highly satisfactory when taking into account the uncertainties related to the measurements and the calculation method.

\section{SEL Hardness AsSURANCE IMPLiCATIONS}

In order to evaluate the impact of using monoenergetic tests results saturated at relatively low energies (e.g. 230 $\mathrm{MeV}$ ) to estimate the operational SEL failure rate for hard environments, the normalized SEL cross section for SRAM C is plotted in Fig. 11 as a function of the environment hardness for the different test locations at CHARM. As can be seen, using the saturated model to evaluate the SEL failure rate in an environment such as those at TL16 and TL17 in CHARM (representative of some locations in the high-energy accelerator context) would lead to an underestimation of roughly a factor 5 and 15 respectively. If the experimental result from a soft spectrum such as TL1 at CHARM or VESUVIO was used instead, the respective underestimation increases to roughly 20 and 80 for the two considered environments. If, on the other hand, a single monoenergetic experimental value is used to characterize the mixed-field response of hard environments, the resulting underestimation factors are $\sim 7$ for TL16 and $\sim 15$ for TL17 if $230 \mathrm{MeV}$ is considered; and $\sim 20$ and $\sim 45$ respectively if the $100 \mathrm{MeV}$ value is used.

Alternatively, results presented here show that in the case of a spectrum similar to the ground-level one (e.g. TL7 in CHARM or what is expected for ChipIR) the SEL rate for the components with a strong energy dependence (SRAMs C and D) is within a factor 2 of both the $100 \mathrm{MeV}$ and saturated model results (difference between dashed and full curve in Fig. 11 for an $\mathrm{H}_{10 \%}$ value of $540 \mathrm{MeV}$, corresponding to the ground-level case) and a factor 3.5 within the soft spectrum in TL1 at CHARM (difference between the data point for the lowest hardness energy in Fig. 11 and the full curve for $\mathrm{H}_{10 \%}=540 \mathrm{MeV}$ ). Therefore as long as these margins can be tolerated, testing in $100 \mathrm{MeV}$ or soft mixed-field spectra can be considered as representative of the ground-level environment.

For the case of an avionic environment (12 km altitude), the saturated model underestimates the expected value of the energy dependent model up to $\mathrm{GeV}$ energies by roughly a factor 4. For a soft mixed-field spectrum such as TL1 for CHARM, the underestimation is roughly a factor 10 . 


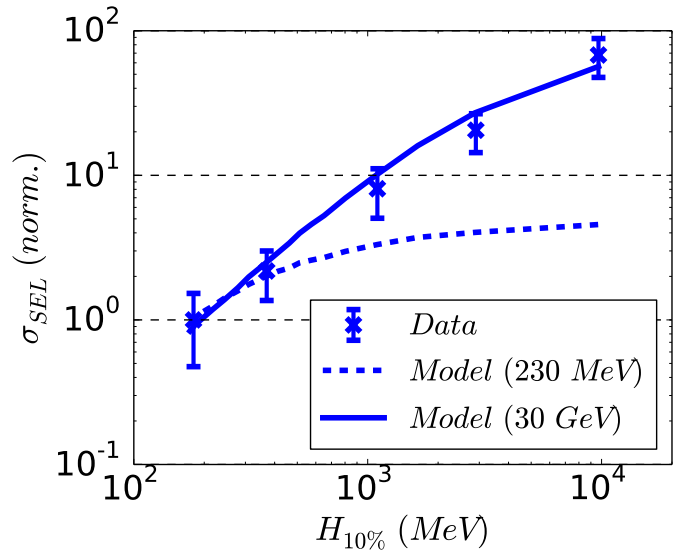

Fig. 11: Experimental mixed-field SEL cross section for SRAM C as a function of the $10 \%$ hardness energy normalized to the value at TL1 in CHARM. The expected SEL cross section from the models saturated at $230 \mathrm{MeV}$ and considering the energy dependence up to $30 \mathrm{GeV}$ are also shown.

Finally, it is to be noted that though the safety margins mentioned in this section apply to a specific set of components and environments, the underlying approach can be used to generalize the results to a broader range of devices and radiation fields as long as sufficient information about the sensitive volume size, materials surrounding it and particle energy spectra is available. If this information is not available, a worst-case dependency can be assumed by considering an SEE cross section fully dominated by tungsten fragments, as was introduced in [3].

\section{CONCLUSIONS AND OUTLOOK}

In this paper we show through experimental and simulated results that not taking the spectral hardness into account for very hard environments can lead to a significant underestimation of the SEL risk. Even for cases in which the goal is not calculating the SEL rate but rather evaluating whether the component is sensitive or not up to a certain fluence level, testing in softer conditions can lead to the erroneous conclusion that the part is SEL free up to a certain radiation level, and then experiencing failure in operation after a significantly lower accumulated fluence. Therefore, in order to obtain realistic failure rate estimations it is important to test the component at a facility which can closely reproduce the operational hadron spectrum. If not possible, the SEL model introduced in [10] or the worst-case factors derived from it as safety margins should be applied to account for the potential impact of the spectral hardness [3]. This conclusion not only applies to the SEL examples shown in this work but also to SEU rates of high critical charge components, owing to the strong contribution of high- $Z$ fragments (as was initially suggested in [24] and later developed in [13] for the case of MBUs). An experimental example of such a case is the radiation hardened component presented in [25].

In addition, the test and model results shown here provide a means of experimentally calibrating any mixed environment in terms of its hardness through measurements using the commercially available components reported in this work that exhibit a strong SEL dependency with energy.

Finally, the CHARM test facility will be used in the future to analyze the mixed-field energy dependence of other destructive events such as SEB in power MOSFETs.

\section{REFERENCES}

[1] P. E. Dodd, M. R. Shaneyfelt, J. R. Schwank, and G. L. Hash, "Neutroninduced latchup in SRAMs at ground level," in Reliability Physics Symposium Proceedings, 2003. 41st Annual. 2003 IEEE International, March 2003, pp. 51-55.

[2] E. Normand, J. L. Wert, P. P. Majewski, D. L. Oberg, W. G. Bartholet, S. K. Davis, M. Shoga, S. A. Wender, and A. Gavron, "Single event upset and latchup measurements in avionics devices using the WNR neutron beam and a new neutron-induced latchup model," in Radiation Effects Data Workshop, 1995, NSREC '95 Workshop Record., 1995 IEEE, 1995, pp. 33-38.

[3] R. G. Alia, E. W. Blackmore, M. Brugger, S. Danzeca, V. FerletCavrois, R. Gaillard, J. Mekki, C. Poivey, K. Roed, F. Saigne, G. Spiezia, M. Trinczek, S. Uznanski, and F. Wrobel, "SEL cross section energy dependence impact on the high energy accelerator failure rate," IEEE Trans. Nucl. Sci., vol. 61, no. 6, pp. 2936-2944, Dec 2014.

[4] Measurement and Reporting of Alpha Particles and Terrestrial Cosmic Ray-Induced Soft Errors in Semiconductor Devices. JEDEC Standard. JESD89A, 2006.

[5] E. W. Blackmore and M. Trinczek, "Intensity Upgrade to the TRIUMF $500 \mathrm{MeV}$ Large-Area Neutron Beam," in Radiation Effects Data Workshop (REDW), 2014 IEEE. IEEE, pp. 1-5.

[6] E. Normand and L. Dominik, "Cross comparison guide for results of neutron SEE testing of microelectronics applicable to avionics," in Radiation Effects Data Workshop (REDW), 2010 IEEE, 2010, pp. 1-8.

[7] F. Miller, C. Weulersse, T. Carriere, N. Guibbaud, S. Morand, and R. Gaillard, "Investigation of $14 \mathrm{MeV}$ neutron capabilities for SEU hardness evaluation," IEEE Trans. Nucl. Sci., vol. 60, no. 4, pp. 2789 2796, Aug 2013.

[8] R. Edwards, C. Dyer, and E. Normand, "Technical standard for atmospheric radiation single event effects, (SEE) on avionics electronics," in Radiation Effects Data Workshop, 2004 IEEE, July 2004, pp. 1-5.

[9] C. Dyer, A. Hands, K. Ford, A. Frydland, and P. Truscott, "Neutroninduced single event effects testing across a wide range of energies and facilities and implications for standards," IEEE Trans. Nucl. Sci., vol. 53, no. 6, pp. 3596-3601, 2006.

[10] R. G. Alia, M. Brugger, S. Danzeca, V. Ferlet-Cavrois, C. Poivey, K. Roed, F. Saigne, G. Spiezia, S. Uznanski, and F. Saigne, "Energy dependence of tungsten-dominated SEL cross sections," IEEE Trans. Nucl. Sci., vol. 61, no. 5, pp. 2718-2726, Oct 2014.

[11] C. Andreani, A. Pietropaolo, A. Salsano, G. Gorini, M. Tardocchi, A. Paccagnella, S. Gerardin, C. D. Frost, S. Ansell, and S. P. Platt, "Facility for fast neutron irradiation tests of electronics at the ISIS spallation neutron source," Applied Physics Letters, vol. 92, no. 11, pp. 114 101114 101-3, Mar 2008.

[12] J. R. Schwank, M. R. Shaneyfelt, J. Baggio, P. E. Dodd, J. A. Felix, V. Ferlet-Cavrois, P. Paillet, D. Lambert, F. W. Sexton, G. L. Hash, and E. W. Blackmore, "Effects of particle energy on proton-induced singleevent latchup," IEEE Trans. Nucl. Sci., vol. 52, no. 6, pp. $2622-2629$, Dec. 2005.

[13] M. A. Clemens, B. D. Sierawski, K. M. Warren, M. H. Mendenhall, N. A. Dodds, R. A. Weller, R. A. Reed, P. E. Dodd, M. R. Shaneyfelt, J. R. Schwank, S. A. Wender, and R. C. Baumann, "The effects of neutron energy and high- $Z$ materials on single event upsets and multiple cell upsets," IEEE Trans. Nucl. Sci., vol. 58, no. 6, pp. 2591 -2598, Dec. 2011.

[14] A. N. Smirnov, A. Pietropaolo, A. V. Prokofiev, E. E. Rodionova, C. D. Frost, S. Ansell, E. M. Schooneveld, and G. Gorini, "Application of thin-film breakdown counters for characterization of neutron field of the VESUVIO instrument at the ISIS spallation source," Nuclear Instruments and Methods in Physics Research A, vol. 687, pp. 14-22, Sep. 2012.

[15] A. Ferrari, P. R. Sala, A. Fasso, and J. Ranft, "FLUKA, a multiparticle transport code," CERN-2005-10-2005, INFN/TC-05/11, SLAC$R-773,2005$.

[16] G. Battistoni, S. Muraro, P. R. Sala, F. Cerruti, A. Ferrari, S. Roesler, A. Fasso, and J. Ranft, "The FLUKA code: description and benchmarking," Proceedings of the Hadronic Shower Simulation Workshop 2006, Fermilab, Sept. 2006 
[17] F. Ballarini, G. Battistoni, M. Brugger, M. Campanella, M. Carboni, F. Cerutti, A. Empl, A. Fasso, A. Ferrari, E. Gadioli, M. Garzelli, M. Lantz, A. Mairani, A. Mostacci, S. Muraro, A. Ottolenghi, V. Patera, M. Pelliccioni, L. Pinsky, J. Ranft, S. Roesler, P. Sala, D. Scannicchio, G. Smirnov, F. Sommerer, S. Trovati, R. Villari, V. Vlachoudis, T. Wilson, and N. Zapp, "The physics of the FLUKA code: Recent developments," Advances in Space Research, vol. 40, no. 9, pp. 1339 - 1349, 2007. [Online]. Available: http://www.sciencedirect.com/science/article/pii/S0273117707004905

[18] F. Lei, S. Clucas, C. Dyer, and P. Truscott, "An atmospheric radiation model based on response matrices generated by detailed Monte Carlo simulations of cosmic ray interactions," IEEE Trans. Nucl. Sci. [Online]. Available: http://www.qarm.eu

[19] K. Roeed, M. Brugger, D. Kramer, P. Peronnard, C. Pignard, G. Spiezia, and A. Thornton, "Method for measuring mixed field radiation levels relevant for SEEs at the LHC," IEEE Trans. Nucl. Sci., vol. 59, no. 4, pp. $1040-1047$, Aug. 2012.

[20] W. Hajdas, F. Burri, C. Eggel, R. Harboe-Sorensen, and R. deMarino, "Radiation effects testing facilities in PSI during implementation of the Proscan project," in Radiation Effects Data Workshop, 2002 IEEE, 2002, pp. $160-164$.

[21] E. W. Blackmore, "Operation of the TRIUMF (20-500 MeV) proton irradiation facility," in Radiation Effects Data Workshop, 2000, 2000, pp. $1-5$.

[22] D. M. Hiemstra and E. W. Blackmore, "LET spectra of proton energy levels from 50 to $500 \mathrm{MeV}$ and their effectiveness for single event effects characterization of microelectronics," IEEE Trans. Nucl. Sci., vol. 50, no. 6, pp. 2245-2250, Dec. 2003.

[23] R. G. Alia, M. Brugger, S. Danzeca, V. Ferlet-Cavrois, C. Poivey, K. Roed, F. Saigne, G. Spiezia, S. Uznanski, and F. Wrobel, "SEE measurements and simulations using mono-energetic GeV-energy hadron beams," IEEE Trans. Nucl. Sci., vol. 60, no. 6, pp. 4142-4149, Dec. 2013.

[24] A. S. Kobayashi, D. R. Ball, K. M. Warren, R. A. Reed, N. Haddad, M. H. Mendenhall, R. D. Schrimpf, and R. A. Weller, "The effect of metallization layers on single event susceptibility," IEEE Trans. Nucl. Sci., vol. 52, no. 6, pp. 2189-2193, Dec 2005.

[25] S. Uznanski, R. G. Alia, E. Blackmore, M. Brugger, R. Gaillard, J. Mekki, B. Todd, M. Trinczek, and A. Villanueva, "The effect of proton energy on SEU cross section of a 16 Mbit TFT PMOS SRAM with DRAM capacitors," IEEE Trans. Nucl. Sci., vol. 61, no. 6, pp. 3074 3079, Dec 2014. 